\title{
Diaphragm and Lung Ultrasound: A Bedside Tool to Hasten Weaning Off from Ventilation
}

\author{
Nitin Dhochak ${ }^{1} \cdot$ Rakesh Lodha $^{1}$ \\ Received: 5 March 2020 / Accepted: 5 March 2020 / Published online: 28 March 2020 \\ (C) Dr. K C Chaudhuri Foundation 2020
}

Failure to wean off mechanical ventilation and need for reintubation adversely affects the clinical outcomes in critically ill children. In a recent study, despite protocolized weaning and spontaneous breathing trials (SBT), $8.3 \%$ and $10.3 \%$ of mechanically ventilated children had extubation failure within $48 \mathrm{~h}$ and need for post extubation non-invasive support, respectively [1]. Extubation failure has been associated with prolonged hospital stay and duration of mechanical ventilation [2]. Diaphragmatic dysfunction has been associated with higher extubation failure rate [2].

Accurate assessment of diaphragmatic function involves special equipment and invasive procedures like esophageal and stomach pressure probes, and phrenic nerve stimulation [1]. Ultrasound can be used as a non-invasive bedside tool to assess diaphragmatic function and lung parenchyma status in critically ill children. Ultrasound can assess percentage change in diaphragmatic thickness from expiration to inspiration (diaphragmatic thickening fraction, DTF) and amplitude of diaphragmatic dome movements in respiratory cycle (diaphragmatic excursion, DE) which are indicators of strength of diaphragmatic contractions [2]. Lung parenchyma is assessed by a semi-quantitative score (Lung Ultrasound Score, LUS) based on frequency of B-lines in different lung fields (score 0 to 3 in each field), with higher score indicating worse parenchymal status [3].

In a study published in this issue of the journal by Abdel Rahman DA et al., 106 mechanically ventilated children were examined with diaphragm and lung ultrasound during SBT [4]. They found that infants with weaning failure had significantly lower DTF and DE, and higher LUS compared to successful weaning ( $p<0.001$ for all comparisons). Performance of the ultrasound parameters was excellent with area under

Rakesh Lodha

rakesh_lodha@hotmail.com

1 Department of Pediatrics, All India Institute of Medical Sciences, New Delhi 110029, India receiver operator curve (AUROC) for DTF, DE and LUS for prediction of weaning failure being $0.932,0.876$ and 0.934 , respectively. Best cut-off values for DTF, DE and LUS for predicting weaning failure were $<23.17 \%,<6.2 \mathrm{~mm}$, and $\geq 12$ respectively. But the current study has unusually high extubation failure rate of $39.6 \%$ [4].

Previous study in 50 children also demonstrated similar cut-off of $\geq 21 \%$ for DTF for successful weaning with successful weaning in $78 \%$ children [2]. Recent meta-analysis of 742 adults reported summary AUROC of 0.838 and 0.859 for DTF and DE, respectively [5]. But cut-offs for DTF and DE were highly variable ranging from 20 to $36 \%$, and 10 to $15 \mathrm{~mm}$, respectively, and there was significant heterogeneity in the studies [5]. A recent multi-centric study including 191 adults older than $65 \mathrm{y}$ with prolonged ventilation $(>7 \mathrm{~d})$ found no difference in mean (SD) DTF and DE in extubation success vs. failure, $29(29) \%$ vs. $38(48) \%(p=0.83)$ and $14(7) \mathrm{mm}$ vs. $11(8) \mathrm{mm}(p=0.13)$ respectively [6]. LUS has not been previously studied in children to predict extubation outcomes. Studies in adults demonstrated significantly higher weaning failure and respiratory distress with higher pre-extubation LUS $[3,7]$.

Both the pediatric studies suggest that weaning outcome can be predicted by ultrasound based diaphragmatic function parameters while results of the large multi-centric study are discouraging for its use in adults $[2,4,6]$. Strength of respiratory efforts are dependent mainly on diaphragm in infants and young children due to their horizontally placed ribs and compliant chest wall, while the contribution of chest wall muscles is greater in adults. This could possibly explain better performance of diaphragmatic ultrasound in infants and young children included in the above pediatric studies.

The study by Abdel Rahman et al. has a few limitations [4]. The authors have not reported the outcome of spontaneous breathing trial (SBT) in the subjects: of 106 children, how many failed a SBT?

Most of the available studies are observational with no comparator arm for estimating added benefit of DTF and DE 
to protocolized extubation with SBT. As success of SBT includes the balance of overall respiratory load and respiratory reserve, it is to be seen if DTF and DE can contribute above that. Though, DTF and DE can be utilized to ascertain respiratory reserve, it will be interesting to see if these parameters could identify candidates for prophylactic non-invasive support post-extubation. Inter-observer variability due to subjective nature of ultrasound reading is one of the limitations. A recent study described inter-rater mean difference in diaphragmatic thickness of $1.3(0.3) \mathrm{mm}$, which is comparable to diaphragmatic thickness in the study by Abdel Rahman et al. [8]. Clinical trials of the ultrasound guided weaning protocols will better clarify its significance in clinical decision making.

\section{Compliance with Ethical Standards}

Conflict of Interest None.

\section{References}

1. Khemani R, Sekayan T, Hotz J, et al. Risk factors for pediatric extubation failure: the importance of respiratory muscle strength. Crit Care Med. 2017;45:e798-805.
2. Xue Y, Zhang Z, Sheng C-Q, Li Y-M, Jia F-Y. The predictive value of diaphragm ultrasound for weaning outcomes in critically ill children. BMC Pulm Med. 2019;19:270.

3. Soummer A, Perbet S, Brisson H, et al. Ultrasound assessment of lung aeration loss during a successful weaning trial predicts postextubation distress. Crit Care Med. 2012;40:2064-72.

4. Abdel Rahman DA, Saber S, El-Maghraby A. Diaphragm and lung ultrasound indices in prediction of outcome of weaning from mechanical ventilation in pediatric intensive care unit. Indian J Pediatr 2020; https://doi.org/10.1007/s12098-019-03177-y.

5. Li C, Li X, Han H, Cui H, Wang G, Wang Z. Diaphragmatic ultrasonography for predicting ventilator weaning: a meta-analysis. Medicine. 2018;97:e10968.

6. Vivier E, Muller M, Putegnat J-B, et al. Inability of diaphragm ultrasound to predict extubation failure: a multicenter study. Chest. 2019;155:1131-9.

7. Soliman SB, Ragab F, Soliman RA, Gaber A, Kamal A. Chest ultrasound in predication of weaning failure. Open Access Maced J Med Sci. 2019;7:1143-7.

8. Ng KWP, Dietz AR, Johnson R, Shoykhet M, Zaidman CM. Reliability of bedside ultrasound of limb and diaphragm muscle thickness in critically ill children. Muscle Nerve. 2019;59:88-94.

Publisher's Note Springer Nature remains neutral with regard to jurisdictional claims in published maps and institutional affiliations. 